\title{
CONVERGENCE RATE TO THE NONLINEAR WAVES FOR VISCOUS CONSERVATION LAWS ON THE HALF LINE*
}

\author{
ITSUKO HASHIMOTO ${ }^{\dagger},{\text { YOSHIHIRO } \text { UEDA }^{\ddagger}, \text { AND SHUICHI KAWASHIMA }}^{\S}$
}

\begin{abstract}
We study the convergence rate of solutions to the initial-boundary value problem for scalar viscous conservation laws on the half line. Especially, we deal with the case where the Riemann problem for the corresponding hyperbolic equation admits transonic rarefaction waves. In this case, it is known that the solution tends toward a linear superposition of the stationary solution and the rarefaction wave. We show that the convergence rate is $(1+t)^{-\frac{1}{2}\left(1-\frac{1}{p}\right)} \log ^{2}(2+t)$ in $L^{p}$ norm $(1 \leq p<\infty)$ and $(1+t)^{-\frac{1}{2}+\epsilon}$ in $L^{\infty}$ norm if the initial perturbation from the corresponding superposition is located in $H^{1} \cap L^{1}$. The proof is given by a combination of the weighted $L^{p}$ energy
\end{abstract} method and the $L^{1}$ estimate.

Key words. Viscous conservation laws, convergence rate, weighted energy method, nonlinear waves, half space.

AMS subject classifications. 35L65, 35F30

1. Introduction. We consider the following scalar viscous conservation laws on the half line:

$$
\begin{cases}u_{t}+f(u)_{x}=u_{x x}, & x>0, t>0, \\ u(0, t)=u_{-}, & t>0, \\ \lim _{x \rightarrow \infty} u(x, t)=u_{+}, & t>0, \\ u(x, 0)=u_{0}(x), & x>0,\end{cases}
$$

where the flux $f=f(u)$ is a given smooth function of $u$ satisfying $f(0)=f^{\prime}(0)=0$ and $u_{ \pm}$are given constants. In this problem, we assume that the initial function $u_{0}(x)$ satisfies $u_{0}(0)=u_{-}$and $\lim _{x \rightarrow \infty} u_{0}(x)=u_{+}$as the compatibility conditions. Throughout this paper, we impose the following condition on the flux $f(u)$ : Either

$$
f^{\prime \prime}(u)>0 \text { for } u \in \mathbb{R},
$$

or

$$
f^{\prime \prime}(0)>0, \quad f(u)>0 \quad \text { for } \quad u \in\left[u_{-}, 0\right) .
$$

The main purpose of the present paper is to obtain the convergence rate under the boundary condition $u_{-}<0<u_{+}$and the flux condition (1.2) or (1.3).

It is known that the asymptotic behavior of solutions to (1.1) is closely related to the solution of the Riemann problem for the corresponding hyperbolic equation (c.f. $[5],[6])$ :

$$
\left\{\begin{array}{l}
u_{t}+f(u)_{x}=0, \quad x \in \mathbb{R}, t>-1 \\
u(x,-1)= \begin{cases}u_{-}, & x<0 \\
u_{+}, & x>0\end{cases}
\end{array}\right.
$$

\footnotetext{
* Received October 2, 2009; accepted for publication November 11, 2009.

${ }^{\dagger}$ Department of Mathematics, Osaka University, Toyonaka, Osaka 560-0043, Japan (i-hashimoto @cr.math.sci.osaka-u.ac.jp).

${ }_{\ddagger}$ Mathematical Institute, Tohoku University, Sendai 980-8578, Japan (ueda@math.tohokuu.ac.jp).

$\S$ Faculty of Mathematics, Kyushu University, Fukuoka 812-8581, Japan (kawashim@math.kyushuu.ac.jp).
} 
In the case where the flux $f(u)$ in (1.1) satisfies the convexity condition (1.2) and the Riemann problem (1.4) has the rarefaction wave solution, Liu-Matsumura-Nishihara [5] showed that the large-time behavior of the solutions depends on the signs of the characteristic speeds $f^{\prime}\left(u_{ \pm}\right)$. More precisely, it is shown that the asymptotic behavior of the solutions in this case is classified into the following three cases:

$$
\begin{array}{lll}
\text { (a) } f^{\prime}\left(u_{-}\right)<f^{\prime}\left(u_{+}\right) \leq 0 & \text { (equivalent to } u_{-}<u_{+} \leq 0 \text { ), } \\
\text { (b) } 0 \leq f^{\prime}\left(u_{-}\right)<f^{\prime}\left(u_{+}\right) & \text {(equivalent to } \left.0 \leq u_{-}<u_{+}\right) \text {, } \\
\text { (c) } f^{\prime}\left(u_{-}\right)<0<f^{\prime}\left(u_{+}\right) & \text {(equivalent to } \left.u_{-}<0<u_{+}\right) .
\end{array}
$$

In the case (a) where $u_{-}<u_{+} \leq 0$, the solutions of (1.1) converge to the stationary solution. Here the stationary solution $\phi(x)$ is defined by the solution of the stationary problem corresponding to (1.1):

$$
\left\{\begin{array}{l}
f(\phi)=\phi_{x}, \quad x>0, \\
\phi(0)=u_{-}, \quad \lim _{x \rightarrow \infty} \phi(x)=u_{+} .
\end{array}\right.
$$

In the case (b) where $0 \leq u_{-}<u_{+}$, the asymptotic state of the solutions is described by the rarefaction wave. Here the rarefaction wave $\psi^{R}(x, t)$ is given as the solution of the Riemann problem (1.4) and is given explicitly for $t>-1$ by

$$
\psi^{R}(x, t)= \begin{cases}u_{-}, & x \leq f^{\prime}\left(u_{-}\right)(t+1), \\ \left(f^{\prime}\right)^{-1}\left(\frac{x}{t+1}\right), & f^{\prime}\left(u_{-}\right)(t+1) \leq x \leq f^{\prime}\left(u_{+}\right)(t+1), \\ u_{+}, & f^{\prime}\left(u_{+}\right)(t+1) \leq x\end{cases}
$$

In the final case (c) where $u_{-}<0<u_{+}$, the asymptotic state of the solutions is given by the superposition of the stationary solution $\phi(x)$ satisfying (1.5) with $u_{+}=0$ and the rarefation wave $\psi^{R}(x, t)$ given by (1.6) with $u_{-}=0$.

There are many papers which discuss the convergence rate toward stationary solutions or rarefaction waves. Harabetian [1] investigated the convergence rate toward the rarefaction waves in the one-dimensional whole space. Kawashima-NishibataNishikawa [3] and Ueda-Nakamura-Kawashima [10] studied the case (a) and obtained the convergence rate toward the corresponding stationary solutions. In particular, the paper [3] treats the non-degenerate case $f^{\prime}\left(u_{+}\right)<0$, while [10] deals with the degenerate case $f^{\prime}\left(u_{+}\right)=0$. On the other hand, the case (b) was considered by Nakamura [8]. He showed the convergence rate toward the corresponding rarefaction waves by using a modified smooth approximation. However, the case (c) has been left open.

Our first purpose of this paper is to obtain the convergence rate in this last case (c) under the convexity assumption (1.2). In this case, as is known, Liu-MatsumuraNishihara [5] already showed the asymptotic stability of the superposition of the stationary solution and the rarefaction wave. More precisely, they proved the following result.

Theorem $1.1([5])$. Suppose that (1.2) and $u_{-}<0<u_{+}$hold. Assume that $u_{0}-u_{+} \in H^{1}$. Then the initial-boundary value problem (1.1) has a unique global solution $u(x, t)$ with $u-u_{+} \in C\left([0, \infty) ; H^{1}\right)$. Moreover, the solution satisfies

$$
\lim _{t \rightarrow \infty} \sup _{x>0}\left|u(x, t)-\phi(x)-\psi^{R}(x, t)\right|=0 .
$$


Here $\phi(x)$ is the stationary solution satisfying (1.5) with $u_{+}=0$ and $\psi^{R}(x, t)$ is the rarefaction wave given by (1.6) with $u_{-}=0$.

Our first main theorem gives the convergence rate for (1.7) and is stated as follows.

Theorem 1.2 (Main Theorem). In addition to the conditions in Theorem 1.1, we assume that $u_{0}-u_{+} \in L^{1}$. Then the solution of the initial-boundary value problem (1.1), which is constructed in Theorem 1.1, satisfies the following quantitative estimates:

$$
\begin{aligned}
& \left\|\left(u-\phi-\psi^{R}\right)(t)\right\|_{L^{p}} \leq C(1+t)^{-\gamma} \log ^{2}(2+t), \\
& \left\|\left(u-\phi-\psi^{R}\right)(t)\right\|_{L^{\infty}} \leq C_{\epsilon}(1+t)^{-\frac{1}{2}+\epsilon}
\end{aligned}
$$

for each $p$ with $1 \leq p<\infty$ and any $\epsilon>0$, where $\gamma=(1 / 2)(1-1 / p)$, and $C$ and $C_{\epsilon}$ are positive constants; $C_{\epsilon}$ is depending on $\epsilon$. Here $\phi(x)$ and $\psi^{R}(x, t)$ are the same as in Theorem 1.1.

On the other hand, the case (c) has also been studied very recently by HashimotoMatsumura [2] when the flux $f(u)$ satisfies the weaker convexity condition (1.3). They proved the asymptotic stability of the superposition of the stationary solution and the rarefaction wave under the smallness condition both on $u_{+}$and the initial perturbation. The result is summarized as follows.

Theorem 1.3 ([2]). Assume (1.3), $u_{-}<0<u_{+}$and $u_{0}-u_{+} \in H^{1}$. Then there is a positive constant $\varepsilon_{0}$ such that, if $u_{+} \leq \varepsilon_{0}$ and $\left\|u_{0}-\phi-\psi^{R}(\cdot, 0)\right\|_{H^{1}} \leq \varepsilon_{0}$, then the initial-boundary value problem (1.1) has a unique global solution $u(x, t)$ with $u-u_{+} \in C\left([0, \infty) ; H^{1}\right)$. Moreover, the solution satisfies the asymptotic relation (1.7).

The second purpose of this paper is to get the convergence rate for (1.7) in Theorem 1.3 under the weaker convexity condition (1.3). Namely, we show the following theorem.

Theorem 1.4 (Main Theorem). In addition to the conditions in Theorem 1.3, we assume that $u_{0}-u_{+} \in L^{1}$. Then there is a positive constant $\varepsilon_{1}$ such that, if $u_{+} \leq \varepsilon_{1}$ and $\left\|u_{0}-\phi-\psi^{R}(\cdot, 0)\right\|_{H^{1}} \leq \varepsilon_{1}$, then the solution of the initial-boundary value problem (1.1), which is constructed in Theorem 1.3, satisfies the quantitative estimates in (1.8).

This paper is organized as follows. In Section 2, we construct a smooth approximation $\psi(x, t)$ of the rarefaction wave $\psi^{R}(x, t)$ in the same way as in [4] and then reformulate our initial-boundary problem (1.1). In Section 3, we consider the convexity case (1.2) and give the proof of Theorem 1.2 by combining the $L^{1}$ estimate and $L^{p}$ estimate for the perturbation. In Section 4 , we treat the weaker convexity case (1.3) and give the proof of Theorem 1.4. This will be done by introducing the special weight function used in [2] and by applying the same argument in Section 3.

Notations. For $1 \leq p \leq \infty, L^{p}=L^{p}(0, \infty)$ denotes the usual Lebesgue space on the half line $(0, \infty)$ with the norm $\|\cdot\|_{L^{p}}$. For an integer $k \geq 0, H^{k}=H^{k}(0, \infty)$ is the $k$-th order $L^{2}$ sense Sobolev space on the half line $(0, \infty)$ with the norm $\|\cdot\|_{H^{k}}$. For an interval $I$ and a Banach space $X, C(I ; X)$ denotes the space of continuous functions on $I$ with values in $X$. Finally, letters $C$ and $c$ in this paper are positive generic constants which may vary from line to line. 
2. Reformulation of the problem. Let $\phi(x)$ be the stationary solution satisfying (1.5) with $u_{+}=0$ and let $\psi^{R}(x, t)$ be the rarefaction wave given by (1.6) with $u_{-}=0$. As in the previous works, we introduce a smooth approximation $\psi(x, t)$ of $\psi^{R}(x, t)$ and define

$$
\Phi(x, t)=\phi(x)+\psi(x, t)
$$

as an approximation of our asymptotic solution $\phi(x)+\psi^{R}(x, t)$. Then we reformulate our problem (1.1) by introducing the perturbation $v(x, t)$ by

$$
u(x, t)=\Phi(x, t)+v(x, t) .
$$

This is the standard strategy for solving our stability problem.

To complete this procedure, we first review the basic properties of the stationary solution $\phi(x)$. For the details, see $[5,6,9]$.

Lemma 2.1. Assume (1.2) or (1.3). Then the stationary problem (1.5) with $u_{+}=0$ has a unique smooth solution $\phi(x)$ satisfying $u_{-}<\phi(x)<0$ and $\phi_{x}(x)>0$ for $x>0$. Moreover, we have

$$
\left|\partial_{x}^{k} \phi(x)\right| \leq C(1+x)^{-k-1}, \quad x \geq 0
$$

for each nonnegative integer $k$.

Next, we introduce a smooth approximation of our rarefaction wave $\psi^{R}(x, t)$. We use the approximation due to Kawashima-Tanaka [4], which is defined by

$$
\psi(x, t)=\left(f^{\prime}\right)^{-1}(\omega(x, t)),
$$

where $\omega(x, t)$ is the smooth solution of the following Riemann problem for the viscous Burgers equation:

$$
\left\{\begin{array}{l}
\omega_{t}+\omega \omega_{x}=\omega_{x x}, \quad x \in \mathbb{R}, \quad t>-1, \\
\omega(x,-1)= \begin{cases}-f^{\prime}\left(u_{+}\right), & x<0, \\
f^{\prime}\left(u_{+}\right), & x>0 .\end{cases}
\end{array}\right.
$$

We note that the Hopf-Cole transformation gives the explicit formula for $\omega(x, t)$. Note also that our approximation $\psi(x, t)$ in $(2.3)$ is well-defined if $f(u)$ is strictly convex on $\left[0, u_{+}\right]$; this is true even in the case (1.3) if $u_{+}$is suitably small. By straightforward computations, we see that our $\psi(x, t)$ satisfies

$$
\psi_{t}+f(\psi)_{x}=\psi_{x x}+\frac{f^{\prime \prime \prime}(\psi)}{f^{\prime \prime}(\psi)} \psi_{x}^{2} .
$$

Let $\psi_{0}(x):=\psi(x, 0)=\left(f^{\prime}\right)^{-1}(\omega(x, 0))$. We summarize the basic properties of $\psi(x, t)$ in the next lemma. For its proof, we refer the reader to [4].

Lemma 2.2. Assume (1.2) or (1.3); in the latter case we assume that $f(u)$ is strictly convex on $\left[0, u_{+}\right]$. Then we have:

1) $\psi(x, t)$ is a smooth solution of (2.5) and verifies $\psi(0, t)=0$ for $t \geq 0$.

2) $0<\psi(x, t)<u_{+}$and $\psi_{x}(x, t)>0$ for $x>0$ and $t \geq 0$. 
3) For $1 \leq p \leq \infty$, we have

$$
\begin{aligned}
& \left\|\psi_{x}(t)\right\|_{L^{p}} \leq C \min \left\{u_{+}(1+t)^{-\gamma}, u_{+}^{1 / p}(1+t)^{-2 \gamma}\right\}, \\
& \left\|\psi_{x x}(t)\right\|_{L^{p}} \leq C \min \left\{u_{+}(1+t)^{-\gamma-\frac{1}{2}},(1+t)^{-\gamma-1}\right\},
\end{aligned}
$$

where $\gamma=(1 / 2)(1-1 / p)$, and $C$ is a constant independent of $u_{+}$.

4) $\psi(x, t)$ is an approximation of $\psi^{R}(x, t)$ in the sense that

$$
\left\|\left(\psi-\psi^{R}\right)(t)\right\|_{L^{p}} \leq C \sigma(t)(1+t)^{-\gamma}
$$

for $1 \leq p \leq \infty$, where $\gamma=(1 / 2)(1-1 / p), \sigma(t)=\log (2+t)$ for $p=1$ and $\sigma(t)=1$ for $1<p \leq \infty$, and $C$ is a constant independent of $u_{+}$.

Now we consider our approximation $\Phi(x, t)$ defined by (2.1). By using (1.5) and (2.5), we see that our $\Phi(x, t)$ satisfies

$$
\Phi_{t}+f(\Phi)_{x}=\Phi_{x x}+h
$$

where

$$
\begin{aligned}
h & =(f(\Phi)-f(\phi)-f(\psi))_{x}+b(\psi) \psi_{x}^{2} \\
& =\left(f^{\prime}(\phi+\psi)-f^{\prime}(\phi)\right) \phi_{x}+\left(f^{\prime}(\phi+\psi)-f^{\prime}(\psi)\right) \psi_{x}+b(\psi) \psi_{x}^{2}
\end{aligned}
$$

with $b(\psi)=f^{\prime \prime \prime}(\psi) / f^{\prime \prime}(\psi)$. Also, we know that $\Phi(0, t)=u_{-}, u_{-}<\Phi(x, t)<u_{+}$and $\Phi_{x}(x, t)>0$ for $x>0$ and $t \geq 0$. Moreover, using the estimates in Lemmas 2.1 and 2.2 , we can estimate the error term $h$ in (2.7) as follows.

Lemma 2.3. We have

$$
\|h(t)\|_{L^{p}} \leq C \min \left\{u_{+}, \sigma(t)(1+t)^{-1}\right\}
$$

for $1 \leq p \leq \infty$, where $\sigma(t)=\log (2+t)$ for $p=1$ and $\sigma(t)=1$ for $1<p \leq \infty$, and $C$ is a positive constant independent of $u_{+}$.

We omit the proof and refer the readers to $[5,2]$.

Now we introduce the perturbation $v(x, t)$ by $(2.2)$ and rewrite our original problem (1.1) as

$$
\left\{\begin{array}{l}
v_{t}+(f(\Phi+v)-f(\Phi))_{x}=v_{x x}-h, \quad x>0, t>0 \\
v(0, t)=0, \quad t>0 \\
v(x, 0)=v_{0}(x), \quad x>0
\end{array}\right.
$$

where we put $v_{0}(x):=u_{0}(x)-\Phi_{0}(x)$ with $\Phi_{0}(x)=\phi(x)+\psi_{0}(x)$. We will discuss this reformulated problem in Sections 3 and 4 to prove our main theorems.

Before closing this section, as a preliminary to our stability analysis, we derive the $L^{1}$ estimate of solutions to the reformulated problem (2.8). To this end, we introduce the functions $s_{\delta}(v)$ and $S_{\delta}(v)$ by

$$
s_{\delta}(v):=\left(\rho_{\delta} * \operatorname{sgn}\right)(v)=\int_{-\infty}^{\infty} \rho_{\delta}(v-y) \operatorname{sgn}(y) d y, \quad S_{\delta}(v):=\int_{0}^{v} s_{\delta}(\eta) d \eta
$$


where the function $\operatorname{sgn}(v)$ is the usual signature function defined by

$$
\operatorname{sgn}(v):=\left\{\begin{array}{cc}
-1 & \text { for } \quad v<0 \\
0 & \text { for } \quad v=0 \\
1 & \text { for } \quad v>0
\end{array}\right.
$$

and $\rho_{\delta} *$ denotes the Friedrichs mollifier. Namely, $\rho_{\delta}(v)=\delta^{-1} \rho(v / \delta)$ with $\rho(v)$ being a nonnegative function satisfying $\rho \in C_{0}^{\infty}(\mathbb{R})$ and $\int_{-\infty}^{\infty} \rho(v) d v=1$. Note that $s_{\delta}(v) \rightarrow$ $\operatorname{sgn}(v)$ and $S_{\delta}(v) \rightarrow|v|$ as $\delta \rightarrow 0$. Also, we have $s_{\delta}(0)=0$ and $s_{\delta}^{\prime}(v)=2 \rho_{\delta}(v) \geq 0$. Making use of these functions, we obtain the following $L^{1}$ estimate of solutions to the problem (2.8).

Proposition 2.4 ( $L^{1}$ estimate). Suppose that the same assumptions in Theorem 1.2 or 1.4 hold true. Then, the solution of (2.8) satisfies the following $L^{1}$ estimate:

$$
\|v(t)\|_{L^{1}} \leq C\left(\left\|v_{0}\right\|_{L^{1}}+1\right) \log ^{2}(2+t),
$$

where $C$ is a positive constant.

Proof. We multiply the equation in $(2.8)$ by $s_{\delta}(v)$. Then a technical computation gives

$$
S_{\delta}(v)_{t}+\left(F^{\delta}-s_{\delta}(v) v_{x}\right)_{x}+s_{\delta}^{\prime}(v) v_{x}^{2}+G^{\delta}=-s_{\delta}(v) h,
$$

where

$$
\begin{aligned}
& F^{\delta}=(f(\Phi+v)-f(\Phi)) s_{\delta}(v)-\int_{0}^{v}(f(\Phi+\eta)-f(\Phi)) s_{\delta}^{\prime}(\eta) d \eta \\
& G^{\delta}=\int_{0}^{v}\left(f^{\prime}(\Phi+\eta)-f^{\prime}(\Phi)\right) s_{\delta}^{\prime}(\eta) d \eta \Phi_{x} .
\end{aligned}
$$

Here we have used the fact that $s_{\delta}(v)(f(\Phi+v)-f(\Phi))_{x}=F_{x}^{\delta}+G^{\delta}$. We integrate $(2.11)$ over $(0, \infty) \times(0, t)$ and take the limit as $\delta \rightarrow 0$. Since $s_{\delta}^{\prime}(v) \geq 0$ and

$$
\left|\int_{0}^{\infty} G^{\delta} d x\right| \leq C \delta\left\|\Phi_{x}\right\|_{L^{1}} \rightarrow 0
$$

as $\delta \rightarrow 0$, we have

$$
\|v(t)\|_{L^{1}} \leq\left\|v_{0}\right\|_{L^{1}}-\int_{0}^{t} \int_{0}^{\infty} \operatorname{sgn}(v) h d x d \tau
$$

Here the right hand side of (2.12) is estimated by using Lemma 2.3 as

$$
\left|\int_{0}^{t} \int_{0}^{\infty} \operatorname{sgn}(v) h d x d \tau\right| \leq \int_{0}^{t}\|h(\tau)\|_{L^{1}} d \tau \leq C \log ^{2}(2+t) .
$$

Substituting this estimate in (2.12) yields the desired estimate (2.10). This completes the proof. $\mathrm{Q}$ 
3. Strictly convex case. In this section, we consider the case where $f(u)$ satisfies (1.2) and prove Theorem 1.2 by applying the time-weighted $L^{p}$ energy method.

Proposition 3.1 (decay estimate). Suppose that the same assumptions in Theorem 1.2 hold true. Then, the solution of (2.8) satisfies the following energy inequalities:

$$
\begin{aligned}
& (1+t)^{\alpha}\|v(t)\|_{L^{p}}^{p}+\int_{0}^{t}(1+\tau)^{\alpha}\left(\left\|\left(|v|^{p / 2}\right)_{x}(\tau)\right\|_{L^{2}}^{2}+\left\|\left(\Phi_{x}^{1 / p} v\right)(\tau)\right\|_{L^{p}}^{p}\right) d \tau \\
& \leq C\left\|v_{0}\right\|_{L^{p}}^{p}+C M^{p}(1+t)^{\alpha-\frac{p-1}{2}} \log ^{2 p}(2+t)
\end{aligned}
$$

for $2 \leq p<\infty$ and $\alpha>(p-1) / 2$, and

$$
\begin{aligned}
& (1+t)^{\alpha}\left\|v_{x}(t)\right\|_{L^{2}}^{2}+\int_{0}^{t}(1+\tau)^{\alpha}\left\|v_{x x}(\tau)\right\|_{L^{2}}^{2} d \tau \\
& \leq C\left\|v_{0}\right\|_{H^{1}}^{2}+C M^{2}(1+t)^{\alpha-\frac{1}{2}} \log ^{4}(2+t)
\end{aligned}
$$

for $\alpha>1 / 2$, where $M=\left\|v_{0}\right\|_{L^{1}}+1$, and $C$ is a positive constant.

Proof. The proof is based on the time-weighted $L^{p}$ energy method in [3], which makes use of certain special interpolation inequalities. We first show the estimate (3.1). We multiply the equation in (2.8) by $|v|^{p-2} v$, where $2 \leq p<\infty$, and compute similarly as in the derivation of (2.11), obtaining

$$
\left(\frac{1}{p}|v|^{p}\right)_{t}+\left(F-|v|^{p-2} v v_{x}\right)_{x}+\frac{4(p-1)}{p^{2}}\left|\left(|v|^{p / 2}\right)_{x}\right|^{2}+G=-|v|^{p-2} v h,
$$

where

$$
\begin{aligned}
& F=(f(\Phi+v)-f(\Phi))|v|^{p-2} v-(p-1) \int_{0}^{v}(f(\Phi+\eta)-f(\Phi))|\eta|^{p-2} d \eta, \\
& G=(p-1) \int_{0}^{v}\left(f^{\prime}(\Phi+\eta)-f^{\prime}(\Phi)\right)|\eta|^{p-2} d \eta \Phi_{x} .
\end{aligned}
$$

Here, by using the strict convexity (1.2) of $f(u)$, we can estimate the term $G$ as

$$
G \geq(p-1) \nu_{0} \int_{0}^{v}|\eta|^{p-2} \eta d \eta \Phi_{x} \geq \frac{p-1}{p} \nu_{0}|v|^{p} \Phi_{x},
$$

where $\nu_{0}$ is a positive constant satisfying $f^{\prime \prime}(u) \geq \nu_{0}$. Now we integrate (3.3) with respect to $x$ over $(0, \infty)$, obtaining

$$
\frac{d}{d t}\|v\|_{L^{p}}^{p}+c_{0}\left(\left\|\left(|v|^{p / 2}\right)_{x}\right\|_{L^{2}}^{2}+\left\|\Phi_{x}^{1 / p} v\right\|_{L^{p}}^{p}\right) \leq p\|v\|_{L^{\infty}}^{p-1}\|h\|_{L^{1}},
$$

where $c_{0}=\min \left\{4(p-1) / p,(p-1) \nu_{0}\right\}$. Then, multiplying (3.4) by $(1+t)^{\alpha}$ with $\alpha>0$ and integrating over $(0, t)$, we have

$$
\begin{aligned}
& (1+t)^{\alpha}\|v(t)\|_{L^{p}}^{p}+c_{0} \int_{0}^{t}(1+\tau)^{\alpha}\left(\left\|\left(|v|^{p / 2}\right)_{x}(\tau)\right\|_{L^{2}}^{2}+\left\|\left(\Phi_{x}^{1 / p} v\right)(\tau)\right\|_{L^{p}}^{p}\right) d \tau \\
& \leq\left\|v_{0}\right\|_{L^{p}}^{p}+\alpha \int_{0}^{t}(1+\tau)^{\alpha-1}\|v(\tau)\|_{L^{p}}^{p} d \tau+p \int_{0}^{t}(1+\tau)^{\alpha}\|v(\tau)\|_{L^{\infty}}^{p-1}\|h(\tau)\|_{L^{1}} d \tau
\end{aligned}
$$


In order to show the desired estimate (3.1), we make use of the following special interpolation inequalities:

$$
\begin{gathered}
\|v\|_{L^{p}} \leq C\left\|\left(|v|^{p / 2}\right)_{x}\right\|_{L^{2}}^{\frac{2(p-1)}{p(p+1)}}\|v\|_{L^{1}}^{\frac{2}{p+1}}, \\
\|v\|_{L^{\infty}} \leq C\left\|\left(|v|^{p / 2}\right)_{x}\right\|_{L^{2}}^{\frac{2}{p+1}}\|v\|_{L^{1}}^{\frac{1}{p+1}}
\end{gathered}
$$

for $2 \leq p<\infty$. Once this is true, we can estimate the second and the third terms on the right hand side of (3.5) as

$$
\begin{aligned}
& \alpha \int_{0}^{t}(1+\tau)^{\alpha-1}\|v(\tau)\|_{L^{p}}^{p} d \tau \leq C \int_{0}^{t}(1+\tau)^{\alpha-1}\left\|\left(|v|^{p / 2}\right)_{x}(\tau)\right\|_{L^{2}}^{\frac{2(p-1)}{p+1}}\|v(\tau)\|_{L^{1}}^{\frac{2 p}{p+1}} d \tau \\
& =C \int_{0}^{t}(1+\tau)^{\alpha}\left\{\left\|\left(|v|^{p / 2}\right)_{x}(\tau)\right\|_{L^{2}}^{2}\right\}^{\frac{p-1}{p+1}}\left\{(1+\tau)^{-\frac{p+1}{2}}\|v(\tau)\|_{L^{1}}^{p}\right\}^{\frac{2}{p+1}} d \tau \\
& \leq \varepsilon \int_{0}^{t}(1+\tau)^{\alpha}\left\|\left(|v|^{p / 2}\right)_{x}(\tau)\right\|_{L^{2}}^{2} d \tau+C_{\varepsilon} \int_{0}^{t}(1+\tau)^{\alpha-\frac{p+1}{2}}\|v(\tau)\|_{L^{1}}^{p} d \tau \\
& p \int_{0}^{t}(1+\tau)^{\alpha}\|v(\tau)\|_{L^{\infty}}^{p-1}\|h(\tau)\|_{L^{1}} d \tau \\
& \leq C \int_{0}^{t}(1+\tau)^{\alpha}\left\|\left(|v|^{p / 2}\right)_{x}(\tau)\right\|_{L^{2}}^{\frac{2(p-1)}{p+1}}\|v(\tau)\|_{L^{1}}^{\frac{p-1}{p+1}}\|h(\tau)\|_{L^{1}} d \tau \\
& =C \int_{0}^{t}(1+\tau)^{\alpha}\left\{\left\|\left(|v|^{p / 2}\right)_{x}(\tau)\right\|_{L^{2}}^{2}\right\}^{\frac{p-1}{p+1}}\left\{\|v(\tau)\|_{L^{1}}^{\frac{p-1}{2}}\|h(\tau)\|_{L^{1}}^{\frac{p+1}{2}}\right\}^{\frac{2}{p+1}} d \tau \\
& \leq \varepsilon \int_{0}^{t}(1+\tau)^{\alpha}\left\|\left(|v|^{p / 2}\right)_{x}(\tau)\right\|_{L^{2}}^{2} d \tau+C_{\varepsilon} \int_{0}^{t}(1+\tau)^{\alpha}\|v(\tau)\|_{L^{1}}^{\frac{p-1}{2}}\|h(\tau)\|_{L^{1}}^{\frac{p+1}{2}} d \tau
\end{aligned}
$$

for any $\varepsilon>0$, where we have used the Young inequality. Substituting (3.8) and (3.9) into (3.5) and choosing $\varepsilon>0$ suitably small, we have

$$
\begin{aligned}
& (1+t)^{\alpha}\|v(t)\|_{L^{p}}^{p}+\int_{0}^{t}(1+\tau)^{\alpha}\left(\left\|\left(|v|^{p / 2}\right)_{x}(\tau)\right\|_{L^{2}}^{2}+\left\|\left(\Phi_{x}^{1 / p} v\right)(\tau)\right\|_{L^{p}}^{p}\right) d \tau \\
& \leq C\left\|v_{0}\right\|_{L^{p}}^{p}+C \int_{0}^{t}(1+\tau)^{\alpha-\frac{p+1}{2}}\|v(\tau)\|_{L^{1}}^{p} d \tau+C \int_{0}^{t}(1+\tau)^{\alpha}\|v(\tau)\|_{L^{1}}^{\frac{p-1}{2}}\|h(\tau)\|_{L^{1}}^{\frac{p+1}{2}} d \tau .
\end{aligned}
$$

Finally, by using Lemmas 2.3 and Proposition 2.4, we estimate the integrals on the right hand side of (3.10) as

$$
\begin{aligned}
C \int_{0}^{t}(1+\tau)^{\alpha-\frac{p+1}{2}}\|v(\tau)\|_{L^{1}}^{p} d \tau & \leq C M^{p} \int_{0}^{t}(1+\tau)^{\alpha-\frac{p+1}{2}} \log ^{2 p}(2+\tau) d \tau \\
& \leq C M^{p}(1+t)^{\alpha-\frac{p-1}{2}} \log ^{2 p}(2+t) \\
C \int_{0}^{t}(1+\tau)^{\alpha}\|v(\tau)\|_{L^{1}}^{\frac{p-1}{2}}\|h(\tau)\|_{L^{1}}^{\frac{p+1}{2}} d \tau & \leq C M^{\frac{p-1}{2}} \int_{0}^{t}(1+\tau)^{\alpha-\frac{p+1}{2}} \log ^{\frac{3 p-1}{2}}(2+\tau) d \tau \\
& \leq C M^{\frac{p-1}{2}}(1+t)^{\alpha-\frac{p-1}{2}} \log ^{\frac{3 p-1}{2}}(2+t)
\end{aligned}
$$


for $\alpha>(p-1) / 2$, where $M=\left\|v_{0}\right\|_{L^{1}}+1$. These estimates together with (3.10) gives the desired estimate (3.1). In particular, we have

$$
\|v(t)\|_{L^{p}} \leq C(1+t)^{-\gamma} \log ^{2}(2+t)
$$

for $2 \leq p<\infty$, where $\gamma=(1 / 2)(1-1 / p)$.

Next, we proceed to the estimate of $v_{x}$. We multiply the equation in (2.8) by $-v_{x x}$. The result is written as

$$
\left(\frac{1}{2} v_{x}^{2}\right)_{t}-\left(v_{t} v_{x}\right)_{x}+v_{x x}^{2}=\left\{f^{\prime}(\Phi+v) v_{x}+\left(f^{\prime}(\Phi+v)-f^{\prime}(\Phi)\right) \Phi_{x}+h\right\} v_{x x} .
$$

Integrating this equality with respect to $x$ over $(0, \infty)$, we have

$$
\frac{1}{2} \frac{d}{d t}\left\|v_{x}\right\|_{L^{2}}^{2}+\left\|v_{x x}\right\|_{L^{2}}^{2} \leq C \int_{0}^{\infty}\left(\left|v_{x}\right|+|v| \Phi_{x}+|h|\right)\left|v_{x x}\right| d x .
$$

Here the right hand side of (3.13) is bounded by $\varepsilon\left\|v_{x x}\right\|_{L^{2}}^{2}+C_{\varepsilon}\left(\left\|v_{x}\right\|_{L^{2}}^{2}+\left\|\Phi_{x}^{1 / 2} v\right\|_{L^{2}}^{2}+\right.$ $\left.\|h\|_{L^{2}}^{2}\right)$ for any $\varepsilon>0$, where $C_{\varepsilon}$ is a constant depending on $\varepsilon$. Therefore, taking $\varepsilon>0$ suitably small, we get

$$
\frac{d}{d t}\left\|v_{x}\right\|_{L^{2}}^{2}+\left\|v_{x x}\right\|_{L^{2}}^{2} \leq C\left(\left\|v_{x}\right\|_{L^{2}}^{2}+\left\|\Phi_{x}^{1 / 2} v\right\|_{L^{2}}^{2}+\|h\|_{L^{2}}^{2}\right) .
$$

Now, multiplying (3.14) by $(1+t)^{\alpha}$ with $\alpha>0$ and integrating the resultant inequality over $(0, t)$, we have

$$
\begin{aligned}
& (1+t)^{\alpha}\left\|v_{x}(t)\right\|_{L^{2}}^{2}+\int_{0}^{t}(1+\tau)^{\alpha}\left\|v_{x x}(\tau)\right\|_{L^{2}}^{2} d \tau \\
& \leq\left\|v_{0, x}\right\|_{L^{2}}^{2}+C \int_{0}^{t}(1+\tau)^{\alpha}\left(\left\|v_{x}(\tau)\right\|_{L^{2}}^{2}+\left\|\left(\Phi_{x}^{1 / 2} v\right)(\tau)\right\|_{L^{2}}^{2}+\|h(\tau)\|_{L^{2}}^{2}\right) d \tau \\
& \leq C\left\|v_{0}\right\|_{H^{1}}^{2}+C M^{2}(1+t)^{\alpha-\frac{1}{2}} \log ^{4}(2+t)
\end{aligned}
$$

for $\alpha>1 / 2$, where we have used (3.1) with $p=2$ and Lemma 2.3 in the last inequality. This shows the desired estimate (3.2). In particular, we have

$$
\left\|v_{x}(t)\right\|_{L^{2}} \leq C(1+t)^{-\frac{1}{4}} \log ^{2}(2+t) .
$$

It remains to prove the estimates (3.6) and (3.7). A general form of the inequality (3.6) in the $n$-dimensional space is found in [3]. Here we give a simpler proof in the one-dimensional space. Let $2 \leq p<\infty$. We put $w=|v|^{p / 2}$ in the one-dimensional Sobolev inequality $\|w\|_{L^{\infty}} \leq C\left\|w_{x}\right\|_{L^{2}}^{1 / 2}\|w\|_{L^{2}}^{1 / 2}$, obtaining

$$
\|v\|_{L^{\infty}} \leq C\left\|\left(|v|^{p / 2}\right)_{x}\right\|_{L^{2}}^{1 / p}\|v\|_{L^{p}}^{1 / 2} .
$$

On the other hand, from the interpolation inequality in $L^{p}$ spaces, we have

$$
\|v\|_{L^{p}} \leq\|v\|_{L^{1}}^{1 / p}\|v\|_{L^{\infty}}^{1-1 / p}
$$

We substitute (3.17) into (3.18) and eliminate $\|v\|_{L^{\infty}}$. This yields the inequality (3.6). Also, substituting (3.6) into (3.17), we get the desired inequality (3.7). Thus the proof of Proposition 3.1 is complete. 
Finally in this section, we complete the proof of Theorem 1.2.

Proof of Theorem 1.2. We have already shown the $L^{p}$ estimate (3.11) for $p=1$ and $2 \leq p<\infty$; notice that (2.10) gives (3.11) for $p=1$. The estimate (3.11) holds true also for $1<p<2$. This easily follows from a simple interpolation inequality $\|v\|_{L^{p}} \leq\|v\|_{L^{1}}^{2 / p-1}\|v\|_{L^{2}}^{2(1-1 / p)}$ for $1 \leq p \leq 2$ together with the estimate (3.11) for $p=1$ and $p=2$. Thus we have proved the estimate (3.11) for $1 \leq p<\infty$.

Next we show the $L^{\infty}$ decay estimate

$$
\|v(t)\|_{L^{\infty}} \leq C_{\epsilon}(1+t)^{-1 / 2+\epsilon}
$$

for any $\epsilon>0$, where $C_{\epsilon}$ is a constant depending on $\epsilon$. For this purpose, we use the following interpolation inequality that is a one-dimensional Gagliardo-Nirenberg inequality:

$$
\|v\|_{L^{\infty}} \leq C\left\|v_{x}\right\|_{L^{2}}^{\theta}\|v\|_{L^{q}}^{1-\theta}
$$

for $1 \leq q<\infty$, where $\theta / 2=(1-\theta) / q$ so that $\theta=2 /(q+2)$. Substituting the estimates (3.11) with $p=q$ and (3.16) into (3.20), we get

$$
\begin{aligned}
\|v(t)\|_{L^{\infty}} & \leq C(1+t)^{-\theta / 4} \log ^{2 \theta}(2+t) \cdot(1+t)^{-\frac{1}{2}(1-1 / q)(1-\theta)} \log ^{2(1-\theta)}(2+t) \\
& =C(1+t)^{-\frac{1}{2}(1-\theta)} \log ^{2}(2+t) .
\end{aligned}
$$

Here, for any $\epsilon>0$, we choose $q$ sufficiently large that $\theta / 2=1 /(q+2)<\epsilon$. For this choice of $q,(3.21)$ gives the desired estimate (3.19).

In order to prove (1.8) in Theorem 1.2 , we recall the relation $u-\phi-\psi^{R}=$ $v+\left(\psi-\psi^{R}\right)$, which gives

$$
\left\|\left(u-\phi-\psi^{R}\right)(t)\right\|_{L^{p}} \leq\|v(t)\|_{L^{p}}+\left\|\left(\psi-\psi^{R}\right)(t)\right\|_{L^{p}}
$$

for $1 \leq p \leq \infty$. This inequality together with (3.11) for $1 \leq p<\infty$, (3.19) and (2.6) gives the desired estimate (1.8). Thus the proof of Theorem 1.2 is complete.

4. Non-convex case. In this section, we consider the case where $f(u)$ satisfies (1.3) and prove Theorem 1.4 by applying the space-time-weighted energy method. Since $f^{\prime \prime}(0)>0$ by (1.3), there are positive constants $r_{0}$ and $\nu_{0}$ such that

$$
f^{\prime \prime}(u) \geq \nu_{0} \quad \text { for } \quad|u| \leq r_{0} .
$$

We make use of the weight function $w(u)$ due to Hashimoto-Matsumura [2], which is given by

$$
w(u)=f(u)+\delta g(u) \quad \text { for } \quad u \in\left[u_{-}, r_{0}\right],
$$

where $\delta$ is a small positive number and $g(u)=-u^{2 m}+r_{0}^{2 m}$ with $m$ being a large positive integer. This weight function satisfies the following property. For the details, see $[2]$.

Lemma 4.1 (weight function, [2]). Suppose that $f(u)$ satisfies (1.3) and let $w(u)$ be the weight function defined in (4.1). Then, for suitably small $\delta>0$ and suitably large integer $m$, there is a positive constant $\nu_{1}$ such that

$$
f^{\prime \prime}(u) w(u)-f(u) w^{\prime \prime}(u) \geq \nu_{1}, \quad w(u) \geq \nu_{1}
$$


for $u \in\left[u_{-}, r_{0}\right]$.

We want to evaluate this weight function $w(u)$ at our asymptotic solution $\Phi(x, t)$ defined in (2.1). To this end, we assume that $u_{+} \leq r_{0}$. Then we have $\Phi(x, t) \in\left[u_{-}, r_{0}\right]$ so that $w(\Phi(x, t))$ is well defined and is uniformly positive:

$$
\nu_{1} \leq w(\Phi(x, t)) \leq C_{1},
$$

where $\nu_{1}$ is the constant in Lemma 4.1 and $C_{1}$ is some positive constant. Now we put

$$
v(x, t)=w(\Phi(x, t)) \tilde{v}(x, t)
$$

and rewrite $(2.8)$ as

$$
\left\{\begin{array}{l}
(\tilde{w} \tilde{v})_{t}+(f(\Phi+\tilde{w} \tilde{v})-f(\Phi))_{x}=(\tilde{w} \tilde{v})_{x x}-h, \quad x>0, t>0 \\
\tilde{v}(0, t)=0, \quad t>0 \\
\tilde{v}(x, 0)=\tilde{v}_{0}(x), \quad x>0
\end{array}\right.
$$

where we simply write $\tilde{w}=w(\Phi)$ and put $\tilde{v}_{0}(x)=v_{0}(x) / w\left(\Phi_{0}(x)\right)$. Here, we consider this function $\tilde{v}(x, t)$ in place of the solution $v(x, t)$ of $(2.8)$ and derive its $L^{p}$ decay estimate. To this end, as a preliminary, we establish a generalized energy equality for (4.4). Let $s(\tilde{v})$ be a smooth function of $\tilde{v}$ satisfying $s(0)=0$ and $s^{\prime}(\tilde{v}) \geq 0$ for any $\tilde{v}$. Put

$$
S(\tilde{v})=\int_{0}^{\tilde{v}} s(\eta) d \eta, \quad T(\tilde{v})=\int_{0}^{\tilde{v}} s^{\prime}(\eta) \eta d \eta
$$

Then we know that $S(\tilde{v}) \geq 0$ and $T(\tilde{v}) \geq 0$ for any $\tilde{v}$. Also, we have the simple relation $S(\tilde{v})+T(\tilde{v})=s(\tilde{v}) \tilde{v}$. Our generalized energy equality for (4.4) is then given as follows.

Proposition 4.2 (generalized energy equality). Assume (1.3) and $u_{+} \leq r_{0}$. Let $\tilde{w}=w(\Phi)$ be the weight function defined above, and let $s(\tilde{v}), S(\tilde{v})$ and $T(\tilde{v})$ be the functions in (4.5). Then our $\tilde{v}(x, t)$ satisfying (4.4) verifies the following energy equality.

$$
\begin{aligned}
& \frac{d}{d t} \int_{0}^{\infty} \tilde{w} S(\tilde{v}) d x+\int_{0}^{\infty} \tilde{w} s^{\prime}(\tilde{v}) \tilde{v}_{x}^{2} d x+\int_{0}^{\infty}\left(f^{\prime \prime} w-f w^{\prime \prime}\right)(\Phi) T(\tilde{v}) \Phi_{x} d x \\
& =\int_{0}^{\infty}(-s(\tilde{v})+O(1) T(\tilde{v})) h d x+\int_{0}^{\infty} O\left(|\psi|+\left|\psi_{x}\right|+|\tilde{v}|\right) T(\tilde{v}) \Phi_{x} d x
\end{aligned}
$$

Proof. We multiply the equation in (4.4) by $s(\tilde{v})$. Then, after technical computations, we have

$$
\begin{aligned}
& (\tilde{w} S(\tilde{v}))_{t}+\left\{F-s(\tilde{v})(\tilde{w} \tilde{v})_{x}+\tilde{w}_{x} T(\tilde{v})\right\}_{x} \\
& +\tilde{w} s^{\prime}(\tilde{v}) \tilde{v}_{x}^{2}+\left(\tilde{w}_{t}-\tilde{w}_{x x}\right) T(\tilde{v})+G=-s(\tilde{v}) h,
\end{aligned}
$$

where

$$
\begin{aligned}
& F=(f(\Phi+\tilde{w} \tilde{v})-f(\Phi)) s(\tilde{v})-\int_{0}^{\tilde{v}}(f(\Phi+\tilde{w} \eta)-f(\Phi)) s^{\prime}(\eta) d \eta \\
& G=\int_{0}^{\tilde{v}}\left\{f^{\prime}(\Phi+\tilde{w} \eta)\left(\Phi_{x}+\tilde{w}_{x} \eta\right)-f^{\prime}(\Phi) \Phi_{x}\right\} s^{\prime}(\eta) d \eta .
\end{aligned}
$$


To see this, we first calculate as

$$
\begin{aligned}
& s(\tilde{v})(\tilde{w} \tilde{v})_{t}=s(\tilde{v})\left(\tilde{w} \tilde{v}_{t}+\tilde{w}_{t} \tilde{v}\right) \\
& =\tilde{w} S(\tilde{v})_{t}+\tilde{w}_{t}(S(\tilde{v})+T(\tilde{v}))=(\tilde{w} S(\tilde{v}))_{t}+\tilde{w}_{t} T(\tilde{v}),
\end{aligned}
$$

where we have used the relation $S(\tilde{v})+T(\tilde{v})=s(\tilde{v}) \tilde{v}$. On the other hand, similarly as in the derivation of $(2.11)$, we have $s(\tilde{v})(f(\Phi+\tilde{w} \tilde{v})-f(\Phi))_{x}=F_{x}+G$. Also, we have

$$
s(\tilde{v})(\tilde{w} \tilde{v})_{x x}=\left\{s(\tilde{v})(\tilde{w} \tilde{v})_{x}\right\}_{x}-s^{\prime}(\tilde{v}) \tilde{v}_{x}(\tilde{w} \tilde{v})_{x} .
$$

Moreover, this last term is calculated as

$$
\begin{aligned}
& s^{\prime}(\tilde{v}) \tilde{v}_{x}(\tilde{w} \tilde{v})_{x}=s^{\prime}(\tilde{v}) \tilde{v}_{x}\left(\tilde{w} \tilde{v}_{x}+\tilde{w}_{x} \tilde{v}\right) \\
& =\tilde{w} s^{\prime}(\tilde{v}) \tilde{v}_{x}^{2}+\tilde{w}_{x} T(\tilde{v})_{x}=\tilde{w} s^{\prime}(\tilde{v}) \tilde{v}_{x}^{2}+\left(\tilde{w}_{x} T(\tilde{v})\right)_{x}-\tilde{w}_{x x} T(\tilde{v}) .
\end{aligned}
$$

These observations prove the equality (4.7).

Now, we derive a simpler expression of the term $G$ in (4.7). A simple calculation gives

$$
\begin{aligned}
& f^{\prime}(\Phi+\tilde{w} \eta)\left(\Phi_{x}+\tilde{w}_{x} \eta\right)-f^{\prime}(\Phi) \Phi_{x} \\
& =\left(f^{\prime}(\Phi+\tilde{w} \eta)-f^{\prime}(\Phi)\right) \Phi_{x}+f^{\prime}(\Phi+\tilde{w} \eta) \tilde{w}_{x} \eta \\
& =\left(f^{\prime \prime}(\Phi) \tilde{w} \eta+O\left(\eta^{2}\right)\right) \Phi_{x}+\left(f^{\prime}(\Phi)+O(\eta)\right) \tilde{w}_{x} \eta \\
& =\left(f^{\prime}(\Phi)_{x} \tilde{w}+f^{\prime}(\Phi) \tilde{w}_{x}\right) \eta+O\left(\eta^{2}\right) \Phi_{x}=\left(f^{\prime}(\Phi) \tilde{w}\right)_{x} \eta+O\left(\eta^{2}\right) \Phi_{x},
\end{aligned}
$$

where we have used the fact that $\tilde{w}_{x}=w^{\prime}(\Phi) \Phi_{x}=O(1) \Phi_{x}$. Therefore we have the expression

$$
G=\int_{0}^{\tilde{v}}\left\{\left(f^{\prime}(\Phi) \tilde{w}\right)_{x} \eta+O\left(\eta^{2}\right) \Phi_{x}\right\} s^{\prime}(\eta) d \eta=\left(f^{\prime}(\Phi) \tilde{w}\right)_{x} T(\tilde{v})+O(|\tilde{v}|) T(\tilde{v}) \Phi_{x} .
$$

Consequently, we obtain

$$
\left(\tilde{w}_{t}-\tilde{w}_{x x}\right) T(\tilde{v})+G=\left\{\tilde{w}_{t}-\tilde{w}_{x x}+\left(f^{\prime}(\Phi) \tilde{w}\right)_{x}\right\} T(\tilde{v})+O(|\tilde{v}|) T(\tilde{v}) \Phi_{x} .
$$

Here, by using (2.7), (2.1) and (1.5), we find that

$$
\tilde{w}_{t}-\tilde{w}_{x x}+\left(f^{\prime}(\Phi) \tilde{w}\right)_{x}=\left(f^{\prime \prime} w-f w^{\prime \prime}\right)(\Phi) \Phi_{x}+O\left(|\psi|+\left|\psi_{x}\right|\right) \Phi_{x}+O(1) h .
$$

In fact, using the relations $\tilde{w}_{x x}=w^{\prime}(\Phi) \Phi_{x x}+w^{\prime \prime}(\Phi) \Phi_{x}^{2}$ and $\left(f^{\prime}(\Phi) \tilde{w}\right)_{x}=\left(f^{\prime} w\right)^{\prime}(\Phi) \Phi_{x}$, we can compute as

$$
\begin{aligned}
& \tilde{w}_{t}-\tilde{w}_{x x}+\left(f^{\prime}(\Phi) \tilde{w}\right)_{x} \\
& =w^{\prime}(\Phi)\left(\Phi_{t}-\Phi_{x x}\right)-w^{\prime \prime}(\Phi) \Phi_{x}^{2}+\left(f^{\prime} w\right)^{\prime}(\Phi) \Phi_{x} \\
& =\left\{\left(f^{\prime} w\right)^{\prime}-f^{\prime} w^{\prime}\right)(\Phi) \Phi_{x}-w^{\prime \prime}(\Phi) \Phi_{x}^{2}+w^{\prime}(\Phi) h \\
& =\left(f^{\prime \prime} w-f w^{\prime \prime}\right)(\Phi) \Phi_{x}+\left(f(\Phi)-\Phi_{x}\right) w^{\prime \prime}(\Phi) \Phi_{x}+w^{\prime}(\Phi) h,
\end{aligned}
$$

where we have also used (2.7). Moreover, since $\Phi=\phi+\psi$ and $\phi_{x}=f(\phi)$, we have

$$
f(\Phi)-\Phi_{x}=(f(\phi+\psi)-f(\phi))-\psi_{x}=O\left(|\psi|+\left|\psi_{x}\right|\right) .
$$


These observations prove (4.12).

Finally, substituting (4.11) and (4.12) into (4.7), we arrive at the equality

$$
\begin{aligned}
& (\tilde{w} S(\tilde{v}))_{t}+\left\{F-s(\tilde{v})(\tilde{w} \tilde{v})_{x}+\tilde{w}_{x} T(\tilde{v})\right\}_{x} \\
& +\tilde{w} s^{\prime}(\tilde{v}) \tilde{v}_{x}^{2}+\left(f^{\prime \prime} w-f w^{\prime \prime}\right)(\Phi) T(\tilde{v}) \Phi_{x}=R
\end{aligned}
$$

where $R=-s(\tilde{v}) h+O(1) T(\tilde{v}) h+O\left(|\psi|+\left|\psi_{x}\right|+|\tilde{v}|\right) T(\tilde{v}) \Phi_{x}$. Integrating this equality with respect to $x$ over $(0, \infty)$ yields the desired energy equality (4.6). This completes the proof of Proposition 4.2.

Before deriving the $L^{p}$ decay estimate for (4.4), we recall the uniform $L^{2}$ estimate for (2.8) which was established in [2] under the assumptions in Theorem 1.3:

$$
\|v(t)\|_{H^{1}}^{2}+\int_{0}^{t}\left(\left\|v_{x}(\tau)\right\|_{H^{1}}^{2}+\left\|\Phi_{x}^{1 / 2} v(\tau)\right\|_{L^{2}}^{2}\right) d \tau \leq C\left(\left\|v_{0}\right\|_{H^{1}}^{2}+u_{+}^{1 / 6}\right) .
$$

This uniform estimate implies that the function $\tilde{v}(v, x)$ defined by (4.3) is also small in $L^{\infty}$, provided that $\left\|v_{0}\right\|_{H^{1}}+u_{+}$is suitably small. This fact will be used in showing the following $L^{p}$ decay estimate for (4.4).

Proposition 4.3 (decay estimate). Suppose that the same assumptions in Theorem 1.4 hold true. Let $\tilde{v}(x, t)$ be the function which is defined by (4.3) and satisfies (4.4). Then there is a positive constant $\varepsilon_{2}$ such that if $\left\|v_{0}\right\|_{H^{1}}+u_{+} \leq \varepsilon_{2}$, then we have

$$
\begin{aligned}
& (1+t)^{\alpha}\|\tilde{v}(t)\|_{L^{p}}^{p}+\int_{0}^{t}(1+\tau)^{\alpha}\left(\left\|\left(|\tilde{v}|^{p / 2}\right)_{x}(\tau)\right\|_{L^{2}}^{2}+\left\|\left(\Phi_{x}^{1 / p} \tilde{v}\right)(\tau)\right\|_{L^{p}}^{p}\right) d \tau \\
& \leq C\left\|v_{0}\right\|_{L^{p}}^{p}+C M^{p}(1+t)^{\alpha-\frac{p-1}{2}} \log ^{2 p}(2+t)
\end{aligned}
$$

for $2 \leq p<\infty$ and $\alpha>(p-1) / 2$, where $M=\left\|v_{0}\right\|_{L^{1}}+1$, and $C$ is a positive constant.

REMARK 1. Since $\left|\left(|v|^{p / 2}\right)_{x}\right| \leq C\left(\left|\left(|\tilde{v}|^{p / 2}\right)_{x}\right|+|\tilde{v}|^{p / 2} \Phi_{x}\right)$, we conclude from (4.15) that the solution $v(x, t)$ of (2.8) satisfies the $L^{p}$ estimate (3.1) for $2 \leq p<\infty$ and $\alpha>(p-1) / 2$ even in the weaker convexity case (1.3).

Proof of Proposition 4.3. We use the generalized energy equality (4.6) with $s(\tilde{v})=$ $|\tilde{v}|^{p-2} \tilde{v}$. For this choice of $s(\tilde{v})$, we have $S(\tilde{v})=(1 / p)|\tilde{v}|^{p}$ and $T(\tilde{v})=((p-1) / p)|\tilde{v}|^{p}$. Moreover, applying Lemma 4.1, we have

$$
\tilde{w} s^{\prime}(\tilde{v}) \tilde{v}_{x}^{2} \geq \frac{4(p-1)}{p^{2}} \nu_{1}\left|\left(|\tilde{v}|^{p / 2}\right)_{x}\right|^{2}, \quad\left(f^{\prime \prime} w-f w^{\prime \prime}\right)(\Phi) T(\tilde{v}) \geq \frac{p-1}{p} \nu_{1}|\tilde{v}|^{p} .
$$

Therefore, we have from (4.6) that

$$
\begin{aligned}
& \frac{d}{d t} \int_{0}^{\infty} w(\Phi)|\tilde{v}|^{p} d x+c_{1}\left(\left\|\left(|\tilde{v}|^{p / 2}\right)_{x}\right\|_{L^{2}}^{2}+\left\|\Phi_{x}^{1 / p} \tilde{v}\right\|_{L^{p}}^{p}\right) \\
& \leq C\|\tilde{v}\|_{L^{\infty}}^{p-1}\|h\|_{L^{1}}+C\left(\left\|\left(\psi, \psi_{x}\right)\right\|_{L^{\infty}}+\|\tilde{v}\|_{L^{\infty}}\right)\left\|\Phi_{x}^{1 / p} \tilde{v}\right\|_{L^{p}}^{p},
\end{aligned}
$$

where $c_{1}=\nu_{1} \min \{4(p-1) / p, p-1\}$ and where we have used $|\tilde{v}| \leq C$. Here we have $\left\|\left(\psi, \psi_{x}\right)\right\|_{L^{\infty}} \leq C u_{+}$by Lemma 2.2. Also, by virtue of (4.14), we see that 
$\|\tilde{v}\|_{L^{\infty}} \leq C\left(\left\|v_{0}\right\|_{H^{1}}+u_{+}^{1 / 12}\right)$. Therefore, assuming that both $\left\|v_{0}\right\|_{H^{1}}$ and $u_{+}$are suitably small, we arrive at the inequality

$$
\frac{d}{d t} \int_{0}^{\infty} w(\Phi)|\tilde{v}|^{p} d x+c\left(\left\|\left(|\tilde{v}|^{p / 2}\right)_{x}\right\|_{L^{2}}^{2}+\left\|\Phi_{x}^{1 / p} \tilde{v}\right\|_{L^{p}}^{p}\right) \leq C\|\tilde{v}\|_{L^{\infty}}^{p-1}\|h\|_{L^{1}}
$$

with some positive constant $c$. Multiplying (4.17) by $(1+t)^{\alpha}$ with $\alpha>0$ and integrating over $(0, t)$, we have

$$
\begin{aligned}
& (1+t)^{\alpha}\|\tilde{v}(t)\|_{L^{p}}^{p}+\int_{0}^{t}(1+\tau)^{\alpha}\left(\left\|\left(|\tilde{v}|^{p / 2}\right)_{x}(\tau)\right\|_{L^{2}}^{2}+\left\|\left(\Phi_{x}^{1 / p} \tilde{v}\right)(\tau)\right\|_{L^{p}}^{p}\right) d \tau \\
& \leq C\left\|\tilde{v}_{0}\right\|_{L^{p}}^{p}+C \int_{0}^{t}(1+\tau)^{\alpha-1}\|\tilde{v}(\tau)\|_{L^{p}}^{p} d \tau+C \int_{0}^{t}(1+\tau)^{\alpha}\|\tilde{v}(\tau)\|_{L^{\infty}}^{p-1}\|h(\tau)\|_{L^{1}} d \tau .
\end{aligned}
$$

This is just the same as (3.5). Therefore, applying the same method as in the proof of Proposition 3.1, we reach the desired estimate (4.15). This completes the proof of Proposition 4.3.

Proof of Theorem 1.4. As remarked in Remark 1, we have already proved (3.1) and hence the $L^{p}$ estimate (3.11) for $2 \leq p<\infty$. This together with the $L^{1}$ estimate (2.10) shows that the $L^{p}$ estimate (3.11) holds true also for $1 \leq p<\infty$. Moreover, we can show the estimate (3.2) because the computations in deriving (3.2) in Section 3 are based only on (3.1) with $p=2$ but do not use the strict convexity condition (1.2). Thus we get the estimate (3.16) and hence the $L^{\infty}$ estimate (3.19) just in the same way as in Section 3. This proves Theorem 1.4.

\section{REFERENCES}

[1] E. Harabetian, Rarefactions and large time behavior for parabolic equations and monotone schemes, Comm. Math. Phys., 114 (1988), pp. 527-536.

[2] I. Hashimoto AND A. MAtsumura, Large time behavior of solutions to an initial boundary value problem on the half line for scalar viscous conservation law, Methods Appl. Anal., 14 (2007), pp. 45-60.

[3] S. KaWAshima, S. Nishibata And M. Nishikawa, $L^{p}$ energy method for multi-dimensional viscous conservation laws and application to the stability of planar waves, J. Hyperbolic Differ. Equ., 1 (2004), pp. 581-603.

[4] S. KaWASHIMA AND Y. TANAKA, Stability of rarefaction waves for a model system of a radiating gas, Kyushu J. Math., 8 (2004), pp. 211-250.

[5] T.-P. Liu, A. Matsumura And K. Nishinara, Behaviors of solutions for the Burgers equation with boundary corresponding to rarefaction waves, SIAM J. Math. Anal., 29 (1998), pp. 293-308.

[6] T.-P. LiU And K. Nishinara, Asymptotic behavior for scalar viscous conservation laws with boundary effect, J. Differential Equations, 133 (1997), pp. 296-320.

[7] A. Matsumura and M. MeI, Nonlinear stability of viscous shock profile for a non-convex system of viscoelasticity, Osaka J. Math., 34 (1997), pp. 589-603.

[8] T. Nakamura, Asymptotic decay toward the rarefaction waves of solutions for viscous conservation laws in a one-dimensional half space, SIAM J. Math. Anal., 34 (2003), pp. 13081317.

[9] Y. UEDA, Asymptotic stability of stationary waves for damped wave equations with a nonlinear convection term, Adv. Math. Sci. Appl., 18 (2008), pp. 329-343.

[10] Y. Ueda, T. Nakamura and S. Kawashima, Stability of degenerate stationary waves for viscous gases, to appear in Archive for Rational Mechanics and Analysis. 